\title{
The Fourth Outrage of Man (Is the Turing-Test Still up to Date?)
}

\author{
Dietmar Dietrich ${ }^{1}$, Matthias Jakubec ${ }^{1 *}$, Samer Schaat ${ }^{1}$, Klaus Doblhammer ${ }^{1}$, Georg Fodor ${ }^{2}$, \\ Christian Brandstaetter ${ }^{1}$ \\ ${ }^{1}$ Institute of Computer Technology at Vienna University of Technology, Gußhausstraße 27-29, 1040 Vienna. \\ 2 University of Cape Town, South Africa. \\ * Corresponding author. Email: matthias.jakubec@aon.at \\ Manuscript submitted October 5, 2015; accepted December 30, 2015. \\ doi: 10.17706/jcp.12.2.116-126
}

\begin{abstract}
This paper addresses psychologists-psychoanalysts in particular-and engineers, as well as others with an interest in technology and a proclivity for critical thinking. We hope to grasp the attention of persons who are skeptical or even uneasy with regard to modern electronic machines. It is important to realize that the mathematician and information theoretician Alan Turing was the first to prompt mankind to consider a comparison between intelligent machines and humans from a scientific perspective. His test proposal piqued the interest of engineers as well as philosophers, and has caused many of them to question their concepts to this day. This treatise aims to show that Turing, a scientist rightfully (albeit belatedly) highly esteemed, formulated a fascinating research question which can be legitimately answered only if one deals intensively and seriously with the disciplines of psychoanalysis and computer engineering. The project SiMA (Simulation of Mental apparatus \& Applications) at the Vienna University of Technology assembled a team of scientists who have come to a concise conclusion and formulated an answer to this question. The answer, however, is not what one might expect.
\end{abstract}

Keywords: Turing test, psychoanalysis, computer engineering.

\section{Introduction}

Alan Turing made the world sit up and take notice in 1950 by not only musing about whether computers could one day become as intelligent as humans, but by becoming the first pioneer to state comprehensibly how this development might be validated [1]. His internationally acclaimed and originally purely theoretical test setup has since been empirically verified a number of times [2], [3]. The Turing test provided experts as well as laypersons with the first ever tool allowing the concept of intelligence to seemingly be tested and "measured". It has often been cited as proof that we have finally reached the point where machine intelligence has become comparable to that of man, or might conceivable soon surpass it. This thought is fascinating in a number of ways, and quite frightening to some. Robots will be superior to the species Homo sapiens in the future? They will outlive mankind? Is this not a massive insult to man, the fourth great outrage? ${ }^{1}$ We, the researchers of project SiMA (Simulation of Mental apparatus \& Applications), are also often asked during seminars, conferences and other events whether our product, i.e.

${ }^{1}$ Cf. [4]: Freud cited three great outrages of mankind: (a) the discovery by Copernicus that the earth is not at the center of the universe, (b) Darwin's discovery that man is descended from animals, and (c) the psychological outrage that due to unconscious motives and contents the ego is not the unrestricted sovereign of its own house. 
the simulation program we are developing in SiMA in order to find a model of the brain, will be able to pass the Turing test. Even our undergraduate and $\mathrm{PhD}$ students are asked such questions during their degree examinations. This is reason enough for us to deal with the topic, for upon closer examination the answers are easy to understand and need not be a source of confusion.

\section{Information Scientists vs. Computer Engineers}

At the time when Turing developed his test, all persons who worked on or with computers were referred to as computer engineers. The information scientist, by contrast, was only "invented" much later. The first information technology curricula in Germany were created in the 1960s, e.g. 1967 in Munich and 1969 in Furtwangen and Karlsruhe. By contrast, one of the authors of this paper, Dietmar Dietrich, was appointed Professor of Computer Engineering in the field of electrical engineering - not in information technology - in 1992. Today the scientific fields of computer engineering and information technology must be strictly differentiated in order to properly understand Turing's problem. By modern standards, Turing would have been an information scientist, for the computer engineer must comprehend the machine as a whole, the structure of hardware and software, and particularly the transitions between these two modeling concepts. The information scientist is more involved with the area of algorithms. This represents a crucial point which will be revisited in the following.

\section{The Turing Test}

Turing had developed his test in order to show by way of a thought experiment that machines would likely eventually be able to think in the same sense as humans. Because "thinking" was a largely intangible term (which it remains to this day), Turing suggested the Imitation Game-the term he used for the test he had developed-as a definition of sorts for human (resp. intelligent) thought: One would be able to say that a machine were capable of thinking precisely if it were capable of winning the Imitation Game, i.e. if it could make a human believe for a certain period of time (Turing set this limit at 5 minutes [1]) that it was human itself. In order to eliminate all factors irrelevant to the assessment of intelligence, the test subject (computer or human) would remain hidden from the tester, with the two communicating only through a channel which today we would call e-mail or a chat room. This means that the experiment is a blind one. In Turing's original setting there were two test subjects, a human and a machine. Both would attempt to behave like humans in the chat room. The tester's task was to determine which of the two subjects was the human being (Fig. 1).

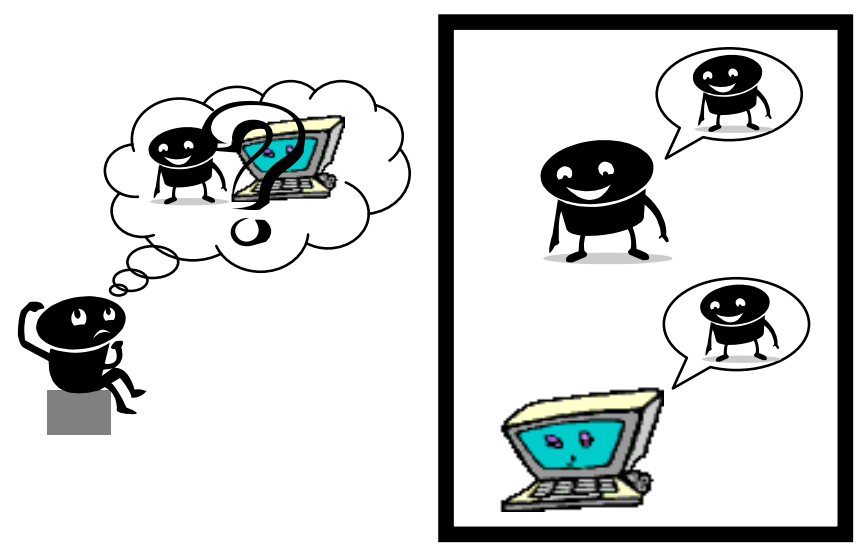

Fig. 1. Turing test.

Turing's specification of his test was obviously rather vague, since at his time machines were nowhere near being able to participate in such an experiment. In the meantime, however, different variants of the 
test exist, as well as programmers who claim that their software is able to pass it. This is in fact true for comparatively simple implementations of the test, but what does that tell us? Only that human testers are easy to fool, for none of the modern software programs which impress us with their "intelligence" are actually doing anything remotely similar to what humans do when they think.

Let us consider test variants which might be slightly more reliable. First off, we must naturally assume double blind experiments. Also, the original test duration of only 5 minutes appears rather short. The test subjects (i.e. the computers or programs being tested) would have to bear up to several different testers including experts like criminologists, psychiatrists or psychotherapists. To purport certain limitations (e.g. a youthful age or psychic ailments) would naturally be forbidden. One could expect such test subjects to have knowledge of the most basic human experiences - like a blind person would know about colors, and in a very literal sense. They would likely know as much about sex as a celibate and speak about sensual experiences like Marcel Proust, for all their knowledge would come exclusively from literature.

\section{Project "Simulating the Mind"}

Project SiMA is currently focused on understanding how the brain functions, i.e. its aim is to develop a model connecting the realm of the neurons (neuronal networks) with the psyche. The project was originally launched by Dietmar Dietrich in 1999 [5], and over the course of time it became apparent that we as researchers were making a number of grave mistakes in the area of artificial intelligence - and repeating them persistently. Today, every scientist knows that one of the fundamental pillars of science is the incorporation of all pertinent research results. This is the reason why project SiMA in its current state has to include not only neurologists but also psychologists, psychoanalysts, information scientists and computer engineers.

A further tragic mistake that is often made is this: In order to achieve a functional model of the brain, it is not sufficient to interpret certain human psychological or neurological phenomena. The development of computer engineering was accompanied by the development of information theory based on natural science. This theory stipulates that an information system which comprehensibly represents the human brain can only be modeled via functional layers. And to that end it is most useful to use a top-down design method. As a consequence, we first require a model of the psyche (Layer 3 (L3), see Fig. 2 (a)) before looking at the circumstances of the neuronal layer (Layer 1 (L1)), for which extensive research results already exist. Armed with this data, we can then model the large and hitherto unspecified Layer 2 (L2) in the layered model as per Fig. 2 (a) using the axiomatic definitions of its interfaces with Layer 1 and Layer 3. The feasibility of this approach was proven in [6].

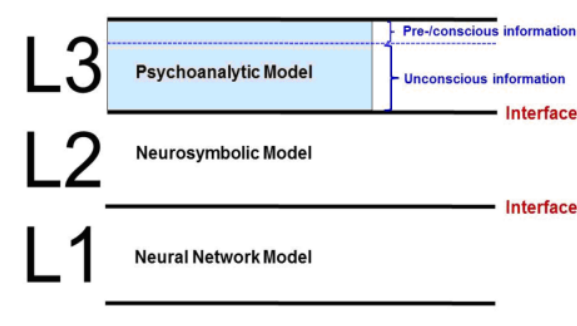

(a)

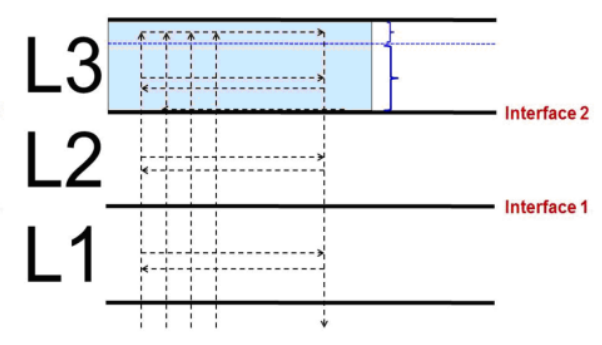

(b)

Fig. 2. (a) Layered model of the brain with the three functional layers L3 to L1; (b) Data flow in the layered model beginning and ending in L1.

The decisive problem at the beginning of project SiMA was the establishment of a model of the psyche. Up until this point, scientists in the field of artificial intelligence had sometimes incorporated aspects from 
brain research and psychology, but interoperability issues between the insights thus gained rarely bothered anyone. And while such research can result in intelligent systems, in whatever way one defines the term, one will certainly not achieve a model of the brain. A protracted search in the course of project SiMA eventually showed clearly that psychoanalytical theory with its first and second topographical model ${ }^{2}$ represents the only practicable functional model of the psyche available today [7]. All other reviewed models were either behavioral models or merely partial models, which are useless to a computer engineer for the purpose of generating a holistic model of the entire system of the brain. Furthermore, the psychoanalytical model, originally devised by the neurologist Sigmund Freud, is functionally compiled - a feature which greatly simplifies the work of the computer engineer. The greatest problem we are still dealing with today is that of axiomatic definitions. Psychoanalysis today is primarily the domain of scientists from the humanities, who have a very different scientific culture from engineers, which is why the members of the SiMA team view themselves as translators-in a quite literal sense-between two cultures [7].

The term complete system as used above requires further explanation. That is, all the functions of the three layers illustrated in Fig. 2(b) must be taken into consideration, since an information flow through them leads to a desired sequence of events in a given scenario. Naturally, this information flow begins in the hardware (sensors like eyes or the tactile sense), then goes through the neurosymbolic layer and is manipulated in the primary process before finally entering the secondary process in which information can become conscious. This is where decisions are made which then travel back through the neurosymbolic layer to the hardware, the muscles and glands. The development process of the model must accordingly occur in steps which allow sound scientific validation. As mentioned above, information theory of computer engineering postulates a top-down design approach for the modeling of information systems. In our case this means that Layers 1 and 2 as per Fig. 2 are initially developed only very rudimentarily, with only Layer 3 , the psyche, being modeled and simulated in detail.

Development begins with a psychoanalytical exemplary case, which includes a description of an exemplary behavior and its interpretation. It is designed by the psychoanalysts in the team since they possess the requisite knowledge. The exemplary case must then be appropriately modified in cooperation with the computer engineers to make it technically implementable. This means that overly complicated relationships must be ignored in the initial iteration. The engineers translate the exemplary case into a technical specification with the help of the psychoanalysts, and the required functions of the psyche eventually result from this specification, leading from Fig. 2 to Fig. 3.

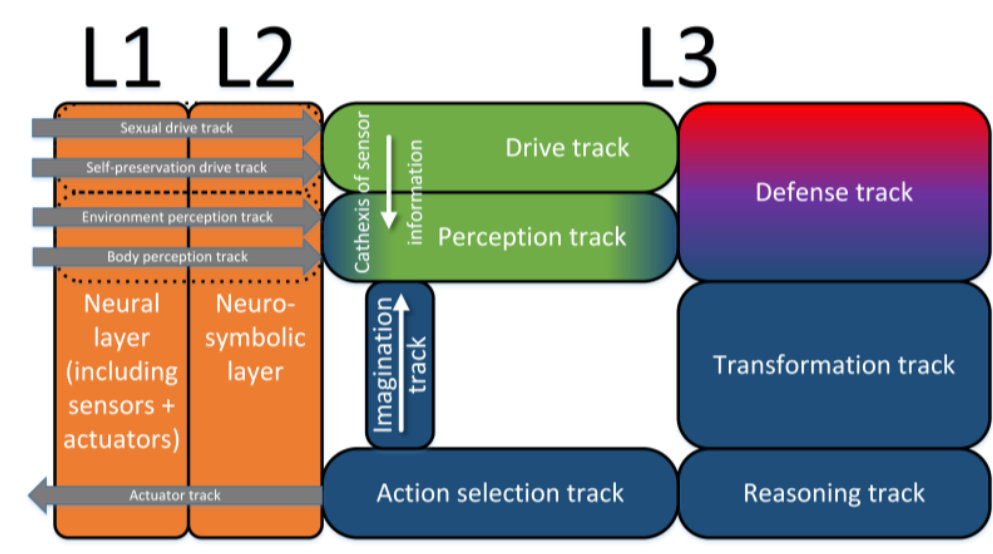

Fig. 3. High-level view on the functions of the brain in the SiMA model, with a focus on the psyche. 
Fig. 3 shows the essential components of the SiMA functional model of the psyche. The psyche (L3) is the third layer, based on the body (neuronal layer L1) and the neurosymbolization layer (L2). Drive impulses from the sexual drive track and the self-preservation track originate in the body and are represented as symbols in the drive track. Bodily sensors allow perception contents from the environment as well as from inside the body to reach the psyche, where they are individually manipulated in the perception track. In addition, information (in psychoanalytical terms: cathexes) from the drive track also reach the perception. The various contents thus generated (in psychoanalytical terms: id-contents, in Fig. 3 green) necessarily come into conflict with each other and with the red field of the super-ego demands, causing the ego (blue) to have to suppress or alter certain contents using the defense mechanisms in the defense track in such a way as to make the contents agreeable for all instances. In the primary-secondary transformation track, the contents allowed to pass are transformed from the primary into the secondary process in order to make them consciousness-capable and compatible with rational and temporal organization. The next step is a pre-conscious focusing on the particular need or wish to be satisfied, which occurs in the reasoning track. Finally, the action to be used for this purpose is chosen and brought into focus in the action selection track, causing it to become conscious. The action track then transmits these intentions back to the body for execution. The various imagined actions that are not selected for execution are transmitted back to the primary process via the imagination track, and affect perception there.

Development of the model illustrated in Fig. 3 has been occurring over the past 15 years in an iterative fashion within project SiMA, with the model being progressively elaborated. The functions shown in Fig. 3 are continually broken down into ever more basic components until they become technically implementable (i.e. programmable). What follows next in each iteration is the simulation of an exemplary case. This involves the need for infusing Simano, the virtual agent with the SiMA brain, with a history and a character. As the psyche of the child is nowhere near as well-researched as that of the grown-up, SiMA assumes a person who is around 30 years old. But how can a history of 30 years be retroactively constructed and integrated into the Simano? We know today that humans cannot forget what has entered into their long-term memory [7]. The amount of information stored by every person in the course of their life is enormous and difficult to even imagine, even for a computer engineer with access to the most modern supercomputers. And what about the psychic functions hitherto not fully researched by psychoanalysis? What of the information flow via hormones in the human body? What about the myriad feedback loops in the various functional layers which are merely hinted at in Fig. 2(b), but are far from being comprehensively addressed? All these factors are inhibitive to "completeness" in the general sense, and of course one could philosophize at length about whether these impediments can ever be overcome. But usual in computer engineering since 1943, when Konrad Zuse developed the first computer, the simulation of a process must always begin with small individual steps before one can begin to deal with the many details. A circuit with the performance of a Zuse computer would be smaller than a dust particle today, but filled a room when it was conceived. Hence in the current context of SiMA, the term "completeness" should be taken to mean what was outlined at the beginning of this chapter, i.e. that in order to simulate a physical process as represented by the human brain, all three layers shown in Fig. 2 must be taken into consideration, even if certain individual functions are present only in a minimal state or not at all.

There is another fact that should be of interest to the knowledgeable reader: Naturally, the SiMA engineers integrate monitors - we refer to them as inspectors - into all of the functions of the psyche to trace how data are handled and changed. This is a first step to allow psychoanalytical theories to be scientifically validated: It is now possible to see what is happening in the functional unit defense or the unit super-ego when information Image 1 comes together with information Image 2 at the point in time t0. For if the Simano in the simulation does not behave as specified in the exemplary-case, then there are only two 
possibilities (disregarding possible programming errors, as finding these is merely a matter of diligence): Either the psychoanalytical theory is incorrect, or it has been misinterpreted and thus incorrectly implemented in the simulation program. This statement is of fundamental significance for project SiMA, and it is with this knowledge that the Turing test can be viewed from a different perspective than those found in many of the reports dealing with it.

\section{Evaluation in the SiMA Project: Is the Model a Plausible Representation of Human Thinking?}

As indicated above, the Turing test inspects a system as a black box: The test is passed if the opposite party (whose nature - human or computer - is unknown), satisfies the expectations of the tester with all their implicit hypotheses ${ }^{3}$. Basically, this is owed to the constrained accessibility of the human psyche, and in this sense the Turing test is based on behavioristic thinking: Behavior patterns which naturally can only be apposite in certain cases are compared.

Psychoanalysis has a contrary approach which is also followed in project SiMA. Here evaluation occurs via case studies, and the simulation allows the thought processes in the Simano, i.e. the artificial psyche, to be examined and tested via the inspectors integrated into the simulation program. This makes it easy to determine whether the thought processes are consistent with the theories of psychoanalysis.

A specific methodology was developed for this purpose in project SiMA [9]: Exemplary cases as employed in casuistry are developed. Functional structures of the psyche are replicated and form the building blocks of the simulation tools, and the question of whether the Simano behaves like a human being from a psychoanalytical point of view is evaluated. To do so, tests are designed with conditions specifying how humans would behave under defined circumstances. These circumstances can be described through four groups of behavioral determinants: First, the current internal state. Second, the external environment. Third, the subject's memories. And fourth, the subject's personality factors ${ }^{4}$. The decision made by a human being at a particular moment is hence determined by these four parameter groups. The concrete values of the determinants are specified with the help of psychoanalysts' experience.

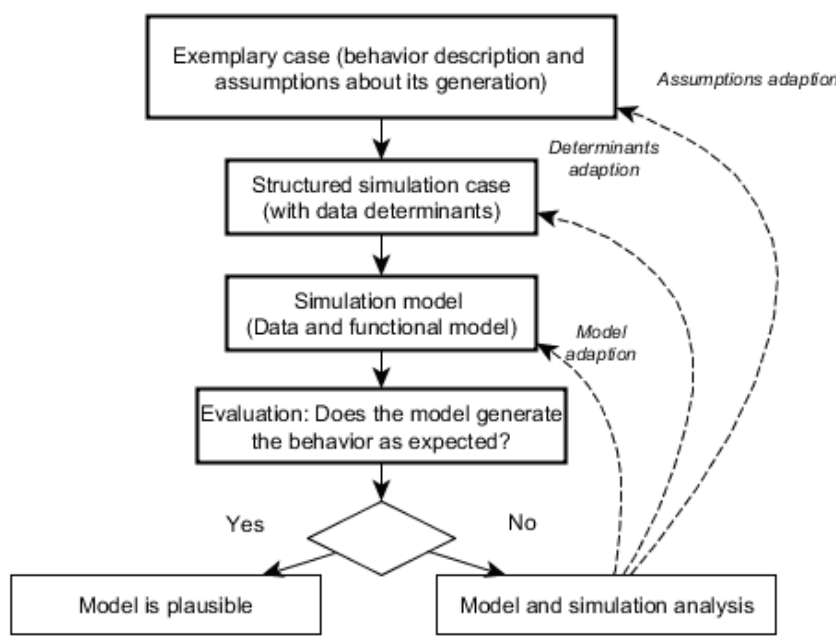

Fig. 4. Testing methodology.

This reveals a fundamental problem: The number of memories collected by a single person over the

${ }^{3}$ Of course the tester has subjective hypotheses on what constitutes a human being. This is a profound point of criticism applicable to the Turing test: The success criteria for the test are imprecise, and therefore inadequately formulated, as seen from a modern psychological perspective.

${ }^{4}$ Personality factors describe bodily circumstances on the one hand, while on the other hand representing simplified representations of personality-defining memories. 
course of their lifetime is nearly infinite. How is it possible to input this amount of information into a computer? Neither can one gather all of the existing memories, nor would it be feasible to input them all if they could be collected. The process must therefore be confined to a small number of basic yet significant events. Which events these are must be determined using the experience of the psychoanalysts designing the exemplary cases.

Hence the exemplary case tests whether the psychoanalytic model assumption generates the expected behavior based on the specified determinants. Of particular interest are observations of changes in the behavior resulting from minor changes to the determinants.

The procedure for creating test routines is illustrated in Fig. 4. The first step is the expertise of psychoanalysts in a casuistic approach in which the behavior triggered by psychic processes is described in a narrative form in exemplary cases. Applied to the Turing test, this would mean the following: What would a human write or answer to a vis-à-vis of which he does not know whether it is a human or a machine? Assumptions are made about the psychic processes and influencing factors that result in this behavior. Our experience in our interdisciplinary work has shown that the psychoanalysts' output requires further structuring and concretization from the perspective of the engineers. Only then can a sufficiently deterministic form be achieved which can be transferred into a simulation. Obviously, this process can be achieved only in iterative sequences between engineers and psychoanalysts.

In contrast to the Turing test, this testing approach allows sufficiently concrete and precise testing thanks in part to the clearly defined success criteria compiled and formulated by scientific experts on the human psyche, i.e. psychoanalysts. Extensive simulations and tests at any desired level of precision can be executed. This is only possible because it is not the behavior that is being modeled and tested, but instead the functions of the psychic model which then indirectly generate the respective behavior. This approach is fundamental for the development of components in modern computer engineering.

\section{Contradictions and Open Issues of the Turing Test}

The outstanding merits of Alan Turing notwithstanding, modern science has undoubtedly progressed beyond his original test method, especially in regard to information theory of computer engineering. When taking into consideration the facts of the psychoanalytical model of the psyche, which Turing was unaware of since he was a mathematician and information scientist in today's terminology, but not a computer engineer (or if he was aware of them, he neglected to incorporate them into his research), it becomes apparent that the Turing test not only represents a goal whose achievement is no longer of significance, but that some of its assumptions are outdated and that as a consequence its entire concept is no longer relevant. It has become a mere vehicle for PR stunts.

There can be no doubt that Turing's original question emphasized the relevance of his thoughts and triggered important scientific questions. We owe it to him that the dualistic way of thinking was finally cast aside in favor of monistic concepts - incidentally, a development which Sigmund Freud also wrestled with. Furthermore, Turing introduced terms like intelligence, consciousness, learning, thinking, etc. into the technical discourse. He used these terms as if they were axiomatically defined, even though as a mathematician he knew that they were not commonly viewed that way. It may thus be assumed that he was being intentionally provocative: Such terms appeal not only to psychology and medicine, but in particular also to philosophy, which is naturally tantalizing coming from a mathematician who would traditionally be expected to deal only with physical and mathematical phenomena.

It is however a different point that will ultimately be decisive. As mentioned above, Sigmund Freud spoke of the three outrages of mankind. That a machine might one day achieve intelligence or even consciousness, and thereby be able to learn and think, must by definition represent the fourth great outrage of man. For 
man has hitherto been the only creature on earth possessing these astounding faculties to such an extent; only humans can feel to such a degree, and plan and act autonomously. But is that really true?

Theories are increasingly being simulated and verified using computer engineering with the help of information science, and this point has now finally also been reached with psychoanalytical theory. The simulated exemplary cases in SiMA scientifically prove that the psychoanalytical theories according to the first and second topographical model are not purely fanciful constructs of ideas, but scientifically attestable models explaining the functions of the psyche and thus human actions and behavior.

But what is the connection to Turing? The Turing test demonstrates a behavior model, but information theory of computer engineering is not interested in comparing behavior. We want to know what functions and history are implemented in the Simano (the virtual agent) as compared to a human being. Though modern robots can ride bicycles like humans, their "thought processes" while doing so are in no way similar to those of humans. The same applies to machines answering questions - as in the case of IBM's Watson [10]. From the point of view of informatics, their algorithmic processes are optimized sequences founded on appropriate mathematical considerations. These considerations have nothing to do, however, with model concepts of the psyche as a whole and its functions, like that offered by psychoanalysis. The computers of today cannot feel - but according to Damasio [11] and Solms [12], feeling is the foundation of all thought. Hence if we wish to develop intelligent machines, we must include the science that has been studying intelligent thought for more than 100 years: psychoanalysis. Through observations of nature, i.e. of human beings-psychoanalysis has systematically established the functions of the human brain and discovered why humans act precisely the way they do.

There is a further problem with behavioral models as compared to functional models. According to Braitenberg [13], the description of a behavior model rises exponentially in complexity compared to the description of the model's functionality. This goes so far that beyond a certain point of complexity the behavior model can no longer be comprehensively described. From this point of view alone there is no sense in describing the human psyche in terms of a behavioral model. The Turing test must therefore be replaced by a function test, and this boils down to the exemplary cases mentioned above which allow the implementations to be appropriately validated.

Let us briefly examine the three other mentioned terms used by Turing: intelligence, learning, and consciousness. The SiMA team relatively quickly came to the realization that intelligence and learning cannot actually be viewed undifferentiatedly. Both intelligence and learning depend on the individual function currently being observed. Learning at the neuronal or neurosymbolic level means something very different from learning in the individual functions of the psyche, and the same applies to intelligence. Learning and intelligence occur in different ways in all the various functions and sub-functions, and their principles in the different functions must not be intermixed or confused with each other. If one nevertheless wishes to examine the overall intelligence of an entire system, then one must do what computer engineering did many years ago: develop benchmark tests which (arbitrarily) define the intelligence of a machine or, respectively, a human. Such a benchmark test for the human brain has already been provided by nature itself in the form of natural selection as defined by Darwin. The same obviously applies to learning.

The terms consciousness and, in particular, feeling are generally somewhat difficult to define and understand. One can however revert to statements by Damasio and Solms, who managed to define both these terms in a way allowing them to be integrated properly into the SiMA model and transduce them into an axiomatically defined scientific model. The problem however lies in the fact that the performance of the functions and in particular the amount of data implemented for the agent's history still need to be greatly increased before this can be validated using an appropriately designed exemplary case. In this regard we 
are still at the beginning, but the results achieved thus far clearly show that we are on the right track - even though that in itself raises a further fundamental question: What constitutes a living entity? Turing did not address this topic, however.

\section{Conclusion}

Turing articulated an important issue indirectly by comparing something previously defined as dead matter with something alive: human beings. This places mankind at an intellectual turning point once again, and once again man must abandon his dreams of being completely unique. Terms like intelligence, learning, consciousness and feeling are not concepts proprietary to mankind alone. They are concepts of nature which upon closer examination can indeed be simulated and therefore also emulated using computers, and hence be implemented e.g. in robots. Turing's considerations need only be adapted to the current state of science, meaning that appropriate exemplary cases must be defined and the theory of psychoanalysis as a whole must be validated. The computer engineering layman should be aware, however, that this process will require many, many more years of work considering that only relatively humble results have been achieved in project SiMA in more than 15 years of intensive research. However, these results are on the verge of being implemented in practice, e.g. in the project ECABA ${ }^{5}$ [15] in the field of energy management or in the project $\operatorname{CogMAS}{ }^{6}[16]$ in the field of purchasing predictions.

\section{References}

[1] A. M. Turing, (1950). Computing machinery and intelligence. Mind, 59, 433-460.

[2] Home page of the loebner prize in artificial intelligence. (2014, September). From http://www.loebner.net/Prizef/loebner-prize.html

[3] Saygin, A. P., Cicekli, I., \& Kman, V. (2000). Turing Test: 50 years later. Minds and Machines 10, 463-518.

[4] Freud, S. (1917). Eine Schwierigkeit der Psychoanalyse. Gesammelte Werke, XII, 3-12.

[5] Dietrich, D. (2000). Evolution potentials for fieldbus systems. Proceedings of IEEE International Workshop on Factory Communication Systems, Instituto Superior de Engenharia do Porto, Portugal.

[6] Velik, R. (2008). A Bionic Model for Human-Like Machine Perception. Dissertation at Institute of Computer Terchnology, University of Technology, Vienna.

[7] Dietrich, D., Fodor, G., Zucker, G., \& Bruckner, D. (2009). Simulating the Mind - A Technical Neuropsychoanalytical Approach, Springer Wien, New York.

[8] List, E. (2009). Psychoanalyse, Vienna.

[9] Dietrich, D., Brandstätter, C., Doblhammer, K., Fittner, M., et al. (December 2014). Naturwissenschaftliches, psychoanalytisches Modell der Psyche für Simulation und Emulation. wissenschaftlicher Bericht, Institute of Computer Technology, Vienna University of Technology.

[10] Schaat, S., \& Dietrich, D. (November 2014). Case-driven agent-based simulation for the development and evaluation of cognitive architectures. Proceedings of 26th Benelux Conference on Artificial Intelligence, (pp. 73-80).

[11] Watson.

From

http://www.research.ibm.com/cognitive-computing/watson/index.shtml\#fbid=9XSWFwxlGRq

[12] Damasio, A. (2004). Ich Fühle, also Bin Ich, Berlin.

[13] Solms, M. Was sind Affekte? Psyche 6/1996, 485-522.

[14] Braitenberg, V. (2004). Vehikel - Experimente mit künstlichen Wesen. Wissenschaftliche Paperbacks, Lit

${ }^{5}$ Energy-efficient Cognitive Autonomous Building Automation

${ }^{6}$ Cognitive Multi-Agent System Supporting Marketing Strategies of Environmental-Friendly Energy Products 
Verlag Münster.

[15] Zucker, G., Habib, U., Blöchle, M., Wendt, A., Schaat, S., \& Siafara, L. (2015). Building energy management and data analytics. Proceedings of 6th Symposium on Communications for Energy Systems, Vienna.

[16] Schaat, S., Miladinovic, A., Wilker, S., Kollmann, S., Dickert, S., Geveze, E., \& Gruber, V. (2015). Emotion in consumer simulations for the development and testing of recommendations for marketing strategies. Proceedings of 3rd Workshop on Emotions and Personality in Personalized Systems, Vienna.

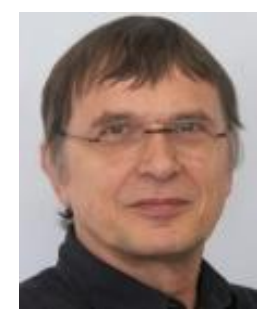

Dietmar Dietrich was born in 1945 at Karlsruhe, Germany, he studied electrical engineering in Karlsruhe and did his Ph.D. in Berlin in the field of control systems.

He is a professor at the Institute of Computer Technology at the Vienna University of Technology and was the head of the institute (about 80 employees) until 2008 since January 1999. He became a full professor of computer technology in 1992.

Dr. Dietrich is a senior member in IEEE, associated editor for Transactions on Industrial Informatics (TII) and Initiator and TC co-chair von BACM (Building Automation, Control and Management Systems) in IES as well. Furthermore he is an associate editor for EURASIP, co-organizer of various tracks and conferences of IEEE.

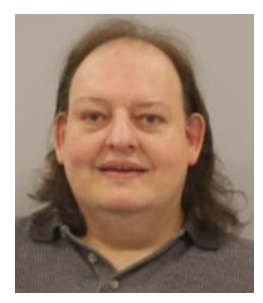

Matthias Jakubec was born in 1964 at Wels, Upper Austria, he studied informatics at the Vienna University of Technology and finished his studies with the master degree of science.

From 1991 to 2010, he worked at Siemens AG Austria, finally as a senior trainer in the field of intelligent networks. Currently he is working on his doctor theses at the Institute of Computer Technology, Vienna University of Technology.

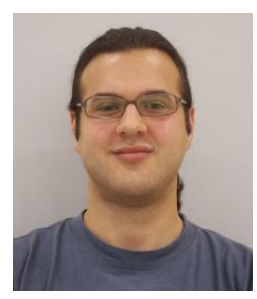

Samer Schaat was born in 1982 in Vienna, Austria, he studied medical informatics at the Vienna University of Technology and finished with the degree of a master of science.

He works as a project leader at the Institute of Computer Technology at the Vienna University of Technology. Currently he is PI for the national funded project Cognitive Multi-Agent System Supporting Marketing Strategies of Environmental-Friendly (CogMAS) and Co-PI for the national funded project Energy-efficient Cognitive Autonomous Building Automation (ECABA).

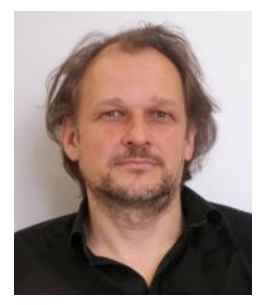

Klaus Doblhammer was born 1966 at Schärding, Austria, he studied physics then pedagogy at University of Vienna and finished pedagogy with the degree of a PhD.

$\mathrm{He}$ worked as a pedagogue for delinquent adolescents and as a supervisor for socialtherapeutic institutions. Currently he is a psychoanalyst in Vienna and a scientific member of the Institute of Computer Technology, Vienna University of Technology.

Dr. Doblhammer is a member of the Vienna Psychoanalytic Association (WAP).

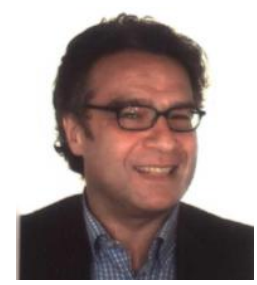

Georg Fodor was born in 1954 at Cluj, Romania. He is a consultant psychiatrist and neurologist, and a psychoanalyst in clinical practice.

He is presently Senior Lecturer at the Department of Neuropsychology, in the Department of Psychology of the University of Cape Town and Department of Neurology of the Groote Schuur Hospital, Cape Town. His main commitment there is the establishment 
of research, teaching and clinical application of Neuropsychoanalysis.

Dr. Fodor is a founding member and board member of the International Neuropsychoanalysis Society; he is a founding member, training analyst, and a board member of the South African Psychoanalytic Association, he is a member of the Vienna Psychoanalytic Association (both Component Societies of the International Psychoanalytic Association); and he is a Teaching Trainer of the Austrian Society for Group Dynamics and Organizational Development.

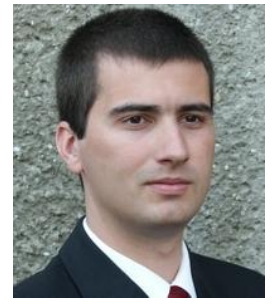

Christian Brandstätter was born in 1979 at Tulln, Austria, he studied informatics and informatics-management at the Vienna University of Technology and finished both studies with the degree master of science.

He works as a system administrator for general IT in a pharmaceutical research institute. His responsibility is the IT infrastructure (network, storage and virtualization). Currently he works on his dissertation and is a member of the Institute of Computer Technology at the Vienna University of Technology. 